\title{
NEUROSCIENCE
}

\section{Optical inhibition in red}

The red light-sensitive optogenetic inhibitor Jaws enables the modulation of neural activity deep inside the brain.

Optogenetics is a powerful approach to manipulate and understand the function of different neurons in the brain. Channelrhodopsin is widely used to activate neurons, but inhibitory tools are less prominent despite their value in showing that the manipulated neurons are required for a certain function or behavior. Ed Boyden and his colleagues

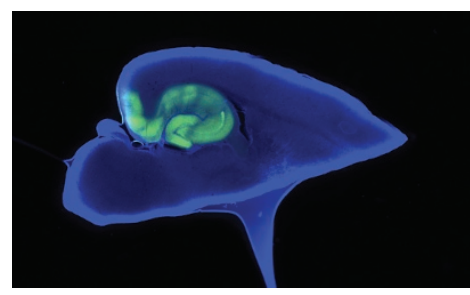

Virus-mediated Jaws expression (green) in the mouse hippocampus. Figure from Chuong et al., Nature Publishing Group. at the Massachusetts Institute of Technology now report a red light-activated chloride pump that effectively inhibits neurons both in vitro and in vivo.

Ideally, optogenetic activators and inhibitors should be activated by red light because it can penetrate tissues more deeply and can even pass through the intact skull. Boyden and his colleagues, led by graduate student Amy Chuong, screened a collection of archaeal halorhodopsins for molecules that are red shifted compared to typical halorhodopsins and that express well in neurons. They found a molecule with the desired properties in the 'Shark' strain of Halobacterium salinarum. They inserted two mutations to increase the light-induced photocurrents and added trafficking sequences to improve its expression in neurons. Aptly, the researchers named the new optogenetic inhibitor Jaws.

Upon stimulation with red light, Jaws leads to a threefold-higher light-induced current in cultured primary neurons compared to previously identified optogenetic inhibitors. This current is mediated by the influx of chloride ions and nearly completely abolishes

\section{SYNTHETIC BIOLOGY}

\section{A PROTEIN CODE TO TARGET RNA}

The protein-RNA specificity code of an RNA-binding protein can be used to target diverse transcripts in the cell for regulation.

The genetic code, by which RNA sequence is translated into protein, was a seminal discovery in molecular biology. In a modern reversal of sorts, Marvin Wickens and researchers in his laboratory at the University of Wisconsin-Madison have uncovered a set of rules that govern how the PUF family of RNA-binding proteins target specific RNA sequences. The code provides unique opportunities to manipulate RNA behavior in living cells.

The Wickens lab codiscovered the second PUF family member through its regulation of a developmental switch in the roundworm. "What was surprising was that these proteins were regulating very different processes," says Wickens, who, along with his University of Wisconsin colleague Judith Kimble, recognized that despite those differences, the PUF proteins share a common mode of action-binding targets to control RNA turnover or translation. Other PUF members have since been implicated in roles spanning development, neurobiology and stem cell biology.

PUF proteins bind each RNA nucleotide via three amino acids located in individual 'PUF repeats' that, in turn, exist in tandem stretches of about eight units. PUFs have been used as tools to target specific RNAs, but efforts to understand specificity have been limited by the need to make many targeted mutations or generate crystal structures. In the Wickens lab, work on the specificity code began as a detour.

"It's kind of a funny story," says Zachary Campbell, lead author of the work. The researchers were interested in how PUF function is affected by protein partners that assemble on RNA, but they became sidetracked when they realized that there was no comprehensive and unbiased way to assess PUF binding affinity. In a collaboration with the neighboring lab of Aseem Ansari, Campbell and the Wickens lab developed the SEQRS method, which uses in vitro selection and high-throughput RNA sequencing to measure 
neural activity in Jaws-expressing neurons. A similarly high inhibition of spontaneous or stimulus-induced neural activity can be achieved when Jaws is expressed in different areas of the mouse cortex and illuminated with red or yellow light. Jaws "has been engineered to be powerful, but we have also validated it to show that it results in powerful silencing in a wide variety of brain regions," says Boyden.

In addition to applications in basic neuroscience research, Jaws has potential for visual therapeutics. Boyden and his collaborators demonstrated this potential by expressing Jaws in cone photoreceptors of a mouse model for retinal degeneration. Healthy cones hyperpolarize in response to light, which can be mimicked by the light-induced chloride currents in Jaws-expressing cells. In the retinal degeneration mouse model, Jaws expression in cone cells restored light-induced activity in downstream ganglion cells.

A complication with optogenetic inhibitors derived from halorhodopsins or archaerhodopsins is their behavior after light is switched off: a rebound burst of action potentials is sometimes observed. "Optogenetic tools are not purely mediating some abstract kind of electricity," says Boyden. Ions are moved across the cell membrane, which can have effects on the cell. To overcome this rebound problem, he and his colleagues have found that gradually decreasing the stimulating light intensity instead of abruptly switching off the light reduces the rebound effect. He thinks that a powerful optogenetic tool can often be made even more powerful by adapting the optical stimulation protocols. In his opinion, "molecular engineering and optical engineering can often go best hand in hand."

Jaws joins an expanding arsenal of tools that allow the optical manipulation and monitoring of neurons. In particular, potentially noninvasive red-shifted tools such as Jaws will be invaluable in our quest to understand how neural circuits function and how they mediate behavior.

Nina Vogt

\section{RESEARCH PAPERS}

Chuong, A.S. et al. Noninvasive optical inhibition with a red-shifted microbial rhodopsin. Nat. Neurosci. 17, 1123-1129 (2014).

the binding of a PUF repeat to up to $4^{20}$ short RNA sequences. In their recent work, they applied the approach to glean the sequence specificity landscapes of 25 different threeamino-acid recognition motifs embedded in a nematode PUF scaffold.

The researchers first used the code to predict the binding specificity of two distantly related PUFs and a well-characterized human PUF; the predictions proved to be very robust. Recognition motifs can be promiscuous, but a few synthetic sequences were more specific than natural motifs. Wickens points out that a typical PUF element binds a sequence the length of a microRNA seed, suggesting off-target binding levels roughly similar to those of microRNAs.

Some gaps in our understanding will take time to fill. For example, the precision of targeting may vary with the scaffold or repeat. "Structure is something I think about," adds Campbell, as SEQRS is performed on RNA segments with minimal secondary structure. But published work has shown that PUFs can bind single-stranded RNA so tightly that they 'melt' double-stranded structures.

The researchers used the code to design a novel sequence, or 'neo-PUF', targeting a transcript encoding the cell division regulator cyclin B1. The neo-PUF was fused to the translation-enhancing yeast poly(A)-binding protein, providing a specific tool to boost translation of cyclin B1 fourfold.

"We need to generate a whole lot more examples of how we can manipulate these proteins," says Campbell. He suggests exploring a wider variety of changes to the PUF repeats and creating new effector proteins that can drive translation in particular compartments such as the neuronal synapse.

"Proteins control virtually everything that happens to RNA," says Wickens. "Processing, transport from the nucleus, and control of stability and movement." It is a great place for biologists interested in regulation to start tinkering.

\section{Tal Nawy}

\section{RESEARCH PAPERS}

Campbell, Z.T. et al. A protein-RNA specificity code enables targeted activation of an endogenous human transcript. Nat. Struct. Mol. Biol. 21, 732-738 (2014). 